\title{
Phonon absorption of far-infrared radiation in small-metal-particle systems
}

\author{
Ping Sheng \\ Exxon Research and Engineering Company, Clinton Township, Route 22 East, Annandale, New Jersey 08801
}

(Received 4 December 1984)

\begin{abstract}
The phonon absorption of small Al spheres is calculated within the jellium model. By taking into account the effect of local jellium-density modulation due to normal modes of elastic oscillations, it is shown that the excitation of a phonon by far-infrared radiation is associated with a transition dipole moment on the order of $10^{-3}$ a.u./sphere. The origin of the dipole, whose magnitude is relatively insensitive to sphere size, can be traced to the incomplete screening of jellium-density modulation near the particle surface. The resulting phonon absorption for a system of $10-\AA \mathrm{Al}$ particles is calculated to be comparable in magnitude to Drude absorption at similar frequencies. Conditions for the experimental observation of the effect are discussed.
\end{abstract}

\section{INTRODUCTION}

Recently the interest in various aspects of small-metalparticle systems, i.e., far-infrared absorption,, ${ }^{1,2}$ surface enhanced Raman scattering, ${ }^{3-5}$ surface effects in optical properties, ${ }^{6}$ etc., has prompted renewed theoretical investigation of their electromagnetic characteristics. In particular, the problem of electronic polarizability and optical response of metal spheres ${ }^{7-10}$ has been the subject of detailed model studies. However, for frequencies in the farinfrared regime, the possibility of phonon absorption ${ }^{11}$ suggests that a complete analysis of the metal-sphere response should include the calculation of ionic polarizability in the presence of free electrons. In this work I use the jellium model to calculate the phonon absorption in the quasistatic limit. It is shown that, in contrast to bulk metal, the phonon absorption by small metal spheres can be fairly appreciable because the sphere surfaces not only break the momentum conservation requirement for absorption transitions (which forbids acoustic phonon absorption in bulk crystals), but also enhance the coupling between the phonon and the electromagnetic radiation through incomplete screening of the surface jelliumdensity modulations arising from elastic vibrations. In what follows, formulation of the density-functional approach is given in Sec. II. Section III presents calculated results together with a discussion of the conditions for the experimental observation of the effect.

\section{FORMULATION}

Consider a sphere of radius $R$ with positively charged uniform jellium density $n_{+}^{0}$ and electron density $n(\mathbf{r})$. The energy of the system $H$ can be written as a function$\mathrm{al}^{12}$ of $n(\mathbf{r})$ :

$$
H[n(\mathbf{r})]=\int d^{3} r\left(E_{\mathrm{kin}}+E_{\mathrm{xc}}+E_{\mathrm{Coul}}\right),
$$

where

$$
\begin{aligned}
& E_{\mathrm{kin}} \equiv a_{1} n^{5 / 3}(\mathbf{r})+a_{2}\left[|\nabla n(\mathbf{r})|^{2} / n(\mathbf{r})\right], \\
& E_{\mathrm{xc}} \equiv-n^{4 / 3}(\mathbf{r})\left\{a_{3}+a_{4} /\left[a_{5}+n^{1 / 3}(\mathbf{r})\right]\right\}, \\
& E_{\mathrm{Coul}} \equiv \frac{1}{2}\left[n(\mathbf{r})-n_{+}^{0}\right] \int d^{3} r^{\prime}\left[n\left(\mathbf{r}^{\prime}\right)-n_{+}^{0}\right] /\left|\mathbf{r}-\mathbf{r}^{\prime}\right|,
\end{aligned}
$$

and $a_{1}=2.8712, \quad a_{2}=\frac{1}{72}, \quad a_{3}=0.7386, \quad a_{4}=0.05641$, $a_{5}=0.07953$, where all quantities are expressed in atomic units (energy unit $=27.2 \mathrm{eV}$, charge unit $=1$ electronic charge, length unit $=0.529 \AA)$. Let $n^{0}(\mathbf{r})$ denote the equilibrium electron-density profile which minimizes $H$. For small disturbances about the equilibrium state, the functional $H[n(\mathbf{r})]$ can be expanded about $n^{0}(\mathbf{r})$ :

$$
H[n(\mathbf{r})]=H\left[n^{0}(\mathbf{r})\right]+\delta H^{(2)}\left[\delta n(\mathbf{r}), n^{0}(\mathbf{r})\right] .
$$

Here $\delta n(\mathbf{r})=n(\mathbf{r})-n^{0}(\mathbf{r})$, and $\delta H^{(2)}$ is a quadratic functional of $\delta n(\mathbf{r})$. The first-order term $\delta H^{(1)}$ in the expansion vanishes because it is given by the expression

$$
\delta H^{(1)}=\int \mu \delta n(\mathbf{r}) d^{3} r,
$$

where $\mu$ is the (constant) chemical potential by definition. Since the number of electrons must remain constant, the integral of $\delta n(\mathbf{r})$ is required to be zero. I assume that the deviation $\delta n(\mathbf{r})$ from the equilibrium profile $n^{0}(\mathbf{r})$ is caused by two sources: a local modulation of the jellium density $Q \Delta_{+}(\mathbf{r})=-n_{+}^{0} \nabla \mathbf{u}(\mathbf{r})$ associated with a given normal mode of elastic oscillation with amplitude $Q$ and displacement field $\mathbf{u}(\mathbf{r})$, and the presence of an external electric field $\epsilon_{0}$ associated with the far-infrared radiation. Since the electron response time is expected to be much shorter than the far-infrared oscillation period of both the electric field and the elastic normal mode, $\Delta_{+}(\mathbf{r})$ and $\epsilon_{0}$ are treated as static quantities in the calculation of $\delta n(\mathbf{r})$. The consideration of elastic oscillations and its coupling to the electron density $n(\mathbf{r})$ gives rise to the following additional energy terms:

$$
\begin{aligned}
& H^{Q}=H_{\text {phonon }}+\delta H_{c}^{Q}, \\
& H_{\text {phonon }}=\frac{1}{2} \sum_{j} m_{j}^{e}\left(\dot{Q}_{j}^{2}+\omega_{j}^{2} Q_{j}^{2}\right), \\
& \delta H_{c}^{Q}=\frac{1}{2} \int d^{3} r d^{3} r^{\prime} \\
& \quad \times \frac{\left[Q^{2} \Delta_{+}(\mathbf{r}) \Delta_{+}\left(\mathbf{r}^{\prime}\right)-2 Q \delta n\left(\mathbf{r}^{\prime}\right) \Delta_{+}(\mathbf{r})\right]}{\left|\mathbf{r}-\mathbf{r}^{\prime}\right|} .
\end{aligned}
$$

Here $H_{\text {phonon }}$ is written in the canonical form for normal modes of elastic oscillations, $m_{j}^{e}$ denotes the effective oscillator mass of the $j$ th normal mode of vibration, $\omega_{j}$ is 
the frequency of the normal mode, and $\delta H_{c}^{Q}$ is obtained by substituting $\left[n_{0}^{+}+Q \Delta_{+}(\mathbf{r})\right]$ for $n_{0}^{+}$in Eq. (1) and collecting terms containing $\Delta_{+}(\mathbf{r})$. The integral of one of the terms, $\Delta_{+}(\mathbf{r})\left[n^{0}\left(\mathbf{r}^{\prime}\right)-n_{+}^{0}\right] /\left|\mathbf{r}-\mathbf{r}^{\prime}\right|$, is noted to be zero in anticipation of the fact that whereas $\left[n^{0}\left(\mathbf{r}^{\prime}\right)-n_{+}^{0}\right]$ has spherical symmetry, the perturbation $\Delta_{+}(\mathbf{r})$ which gives rise to dipole moments and phonon absorption, possesses dipolar symmetry. This accounts for a term's absence in Eq. (3c). In addition to elastic vibrations, the energy due to the presence of electric field can be written in the form

$$
\begin{aligned}
& H^{\epsilon}=H_{\text {photon }}+\delta H_{c}^{\epsilon}, \\
& H_{\text {photon }}=\frac{1}{2} \int \frac{\epsilon_{0}^{2}+H_{0}^{2}}{4 \pi} d^{3} r \\
& \delta H_{c}^{\epsilon}=\epsilon_{0} \cdot \int \mathrm{r}\left[\delta n(\mathbf{r})-Q \Delta_{+}(\mathbf{r})\right] d^{3} r .
\end{aligned}
$$

In the expression for $\delta H_{c}^{\epsilon}$, Eq. (4c), the term involving the integral of $\epsilon_{0} \cdot \mathbf{r}\left[n^{0}(\mathbf{r})-n_{0}^{+}\right]$has been set to zero due to symmetry consideration. The total energy of the system can now be written as

$$
\begin{aligned}
& H_{\text {total }}=H\left[n^{0}(\mathbf{r})\right]+H_{\text {phonon }}+H_{\text {photon }}+\Delta H, \\
& \Delta H=\delta H^{(2)}+\delta H_{c}^{Q}+\delta H_{c}^{\epsilon},
\end{aligned}
$$

where $\Delta H$ is regarded as the perturbation term. Now we recall that $\delta n(\mathbf{r})$ is caused by elastic vibrations and the presence of electric field. For $Q$ and $\epsilon_{0}$ small, linearresponse theory dictates that

$$
\delta n(\mathbf{r})=\delta s(\mathbf{r}) \epsilon_{0}+\delta t(\mathbf{r}) Q,
$$

where $\delta s \epsilon_{0}$ and $\delta t Q$ denote the induced electron-density changes associated with the presence of electric field and jellium-density modulation, respectively. When this expression for $\delta n(\mathbf{r})$ is substituted into $\Delta H$, three types of terms emerge. One type is proportional to $Q^{2}$ and represents that part of the phonon energy associated with compressing a plasma. This type of term can be renormalized away and regarded as part of $H_{\text {phonon }}$, with $\omega_{j}$ modified to include the additional contribution. The second type of term is proportional to $\epsilon_{0}^{2}$ and represents the classical interaction energy between the electric field and the polarizable sphere (with a rigid core). The third type, proportional to $\epsilon_{0} Q$, represents the interaction energy between the electric field and the normal mode of elastic oscillations. If we denote this energy as $\delta H_{\text {int }}=-\mathbf{p} \cdot \epsilon_{0}$, then $\left|\mathbf{p}_{j}\right|=K_{j} Q_{j}$ is the effective dipole moment generated by the $j$ th normal mode of vibrations.

Given $\delta H_{\text {int }}$, one can use Fermi's golden rule to calculate the rate of transitions and absorption. In particular, in the noninteracting limit the absorption by individual spheres is additive and the absorption constant $\eta$ is given by

$$
\eta=\frac{8 \pi^{3}}{3} v f \sum_{j} K_{j}^{2}\left|\left\langle b\left|Q_{j}\right| a\right\rangle\right|^{2} \delta\left(\hbar \omega_{b a}-\hbar \omega\right),
$$

where $v=1 / \lambda$ expresses frequency in wave numbers, $\lambda$ is the wavelength, the factor $\frac{1}{3}$ comes from directional averaging of $\mathbf{p}$ relative to $\epsilon_{0}, f$ is the volume fraction of metal spheres, $a$ is the initial state, and $b$ is the final state of transition. In this case, since $Q_{j}$ will only connect ini- tial and final states differing by 1 phonon number of the $j$ th normal mode, the matrix elements can be evaluated from the standard quantization of the phonon (harmonicoscillator) Hamiltonian $H_{\text {phonon }}$ :

$$
\left\langle b\left|Q_{j}\right| a\right\rangle=\frac{1}{i}\left[\frac{\hbar}{2 m_{j}^{e} \omega_{j}}\right]^{1 / 2}(n+1)^{1 / 2},
$$

where $\hbar$ is Planck's constant $h$ divided by $2 \pi, n$ denotes the number of phonons in state $|a\rangle$, and $n+1$ the number of phonons in state $|b\rangle$. Therefore, Eq. (7) can be written as

$$
\eta=\frac{8 \pi^{3}}{3} v f \sum_{j}\left|\mathbf{p}_{j}\right|^{2} \delta\left(\hbar \omega_{j}-\hbar \omega\right),
$$

where

$$
\left|\mathbf{p}_{j}\right|^{2}=\left(\bar{n}_{j}+1\right) \frac{K_{j}^{2} \hbar}{2 m_{j}^{e} \omega_{j}},
$$

and we have used $\bar{n}_{j}=\left[\exp \left(\hbar \omega_{j} / k_{B} T\right)-1\right]^{-1}$ to denote the average phonon occupation number of the $j$ th mode in thermal equilibrium ( $k_{B}$ is Boltzmann's constant, $T$ the temperature). In general, if we have a collection of spheres of various sizes, the effect of local-field correction means that the complex dielectric constant $\epsilon$ of the composite is given by ${ }^{13}$

$$
\begin{aligned}
\frac{\epsilon-1}{\epsilon+2} & =\frac{1}{3} \int_{0}^{\infty} P(R) \alpha_{R} d R \\
\alpha_{R}= & f \frac{R_{0}^{-3}}{\int_{0}^{\infty} x^{3} P(x) d x} \frac{1}{2 h c} \\
& \times \sum_{j}\left|\mathbf{p}_{j}\right|^{2}\left(\frac{4 v}{v_{j}^{2}-v^{2}}+i 2 \pi \delta\left(v_{j}-v\right)\right) .
\end{aligned}
$$

Here $c$ is speed of light, and $R_{0}$ the most probable radius of the distribution. From the dielectric constant $\epsilon$ the absorption constant $\eta$ can be simply obtained as $4 \pi v \operatorname{Im}\left(\epsilon^{1 / 2}\right)$.

From the above discussion it is seen that the task of calculating the phonon absorption lies essentially in the evaluation of $\left|\mathbf{p}_{j}\right|$. For a given phonon mode and electric field $\epsilon_{0}$, such evaluation requires the knowledge of the electronic response $\delta n(\mathbf{r})$. In the remaining part of this section I describe the procedure by which the calculation of $\delta n(\mathbf{r})$ can be accomplished. First, in order to evaluate the jellium modulation $\Delta_{+}(\mathbf{r})$ due to a vibration mode, it is noted that from general symmetry arguments $\delta H_{\text {int }}$ is nonzero only for those normal modes of oscillation with dipolar angular dependence. Thus the displacement field $\mathbf{u}(\mathbf{r})$ must be of the form ${ }^{14}$

$$
\begin{aligned}
\mathbf{u}(\mathbf{r}) / Q= & \hat{\mathbf{r}}\left[j_{1}^{\prime}\left(k_{n}^{c} r\right)+2 A_{n} j_{1}\left(k_{n}^{s} r\right) /\left(k_{n}^{s} r\right)\right] \cos \theta \\
& -\widehat{\boldsymbol{\theta}}\left\{A_{n}\left[k_{n}^{s} r j_{1}\left(k_{n}^{2} r\right)\right]^{\prime} / k_{n}^{s} r+j_{1}\left(k_{n}^{c} r\right) / k_{n}^{c} r\right\} \sin \theta,
\end{aligned}
$$

where $\hat{\mathbf{r}}$ and $\hat{\boldsymbol{\theta}}$ are unit vectors in the radial and polar angle directions, respectively, $k_{n}^{c(s)}=\omega_{n} / v_{c(s)}$ is the compressional (shear) wave vector for the $n$th eigenmode with frequency $\omega_{n}, v_{c(s)}$ is the compressional (shear) wave speed, 
$j_{1}$ denotes spherical Bessel function of order 1 , and $\left(1, A_{n}\right)$ is the eigenvector associated with the $n$th mode. (Here $n=1,2, \ldots$ indexes the eigenfrequencies in ascending order.) Once $\Delta_{+}(\mathbf{r})$ is obtained as $-n_{0}^{+} \nabla \cdot \mathbf{u}(\mathbf{r}) / Q$, the next step is the calculation of $\delta s$ and $\delta t$ through the principle of energy minimization. Since electron-density perturbation must vanish at the center of the sphere (from dipolar symmetry) and become exponentially small at some suitable distance $(\sim 8$ a.u.) out from the sphere boundary, it is natural to expand $\delta s$ and $\delta t$ in terms of the complete set of functions as

$$
\begin{aligned}
& \delta s(\mathbf{r})=\cos \theta_{1} \sum_{i=1}^{\infty} C_{i} j_{1}\left(\beta_{i} r / a\right), \\
& \delta t(\mathbf{r})=\cos \theta_{2} \sum_{j=1}^{\infty} D_{j} j_{1}\left(\beta_{j} r / a\right),
\end{aligned}
$$

where $a=R+8$ a.u. is the outer radius of the region in which $\delta s$ and $\delta t$ are defined, $\beta_{i}$ 's are the roots of $j_{1}\left(\beta_{i}\right)=0, \theta_{1}$ is the polar angle defined by taking the electric-field direction as the $z$ axis, and $\theta_{2}$ is the polar angle defined relative to the dipole symmetry axis of the elastic oscillation. The angle between the two symmetry directions will be denoted as $\theta_{12}$. Since $\delta s(\mathbf{r}) \epsilon_{0}$ and $\delta t(\mathbf{r}) Q$ are linearly additive, the expansion coefficients $C_{i}$ and $D_{j}$ are determined by the minimization conditions $\partial(\Delta H) /\left.\partial C_{i}\right|_{Q=0}=0$ and $\partial(\Delta H) /\left.\partial D_{j}\right|_{\epsilon_{0}=0}=0$, respectively. The two infinite sets of linear simultaneous equations that result differ only in the forcing terms:

$$
M_{i j} X_{j}=F_{i}^{\epsilon(Q)}
$$

where

$$
\begin{aligned}
& M_{i j}=m_{i j} a^{3}+c_{i j} a^{5}, \\
& m_{i j}=\frac{4 \pi}{3} \cdot \int_{0}^{1} x^{2} d x\left[2 N_{0}(a x) j_{1}\left(\beta_{i} x\right) j_{1}\left(\beta_{j} x\right)+2 N_{1}(a x) \frac{\beta_{i} \beta_{j}}{a^{2}} j_{1}^{\prime}\left(\beta_{i} x\right) j_{1}^{\prime}\left(\beta_{j} x\right)\right. \\
& +N_{2}(a x)\left[\frac{\beta_{i}}{a} j_{1}^{\prime}\left(\beta_{i} x\right) j_{1}\left(\beta_{j} x\right)+\frac{\beta_{j}}{a} j_{1}^{\prime}\left(\beta_{j} x\right) j_{1}\left(\beta_{i} x\right)\right], \\
& c_{i j}=\frac{8 \pi^{2}}{9} \frac{1}{\beta_{j}^{2} \beta_{i}^{2}}\left[\left[\frac{3}{2 \beta_{i}^{4}} \gamma\left(\beta_{i}\right) \delta_{i j}-\sin \beta_{j}\right] \gamma\left(\beta_{i}\right)+\left[\frac{3}{2 \beta_{j}^{4}} \gamma\left(\beta_{j}\right) \delta_{i j}-\sin \beta_{i}\right] \gamma\left(\beta_{j}\right)\right] \text {, } \\
& F_{i}^{\epsilon}=\frac{4 \pi}{3} \frac{\gamma\left(\beta_{i}\right)}{\beta_{i}^{4}} a^{4}, \\
& F_{i}^{Q}=-\frac{16 \pi^{2}}{9} R^{4} \frac{3 \alpha_{n}\left[\sin \alpha_{n} j_{1}\left(\beta_{i} R / a\right)-\sin \left(\beta_{i} R / a\right) j_{1}\left(\alpha_{n}\right)\right]}{\left(\beta_{i} R / a\right)^{2}\left[\left(\beta_{i} R / a\right)^{2}-\alpha_{n}^{2}\right]}+\frac{\sin \beta_{i}\left[\sin \alpha_{n}-3 j_{1}\left(\alpha_{n}\right)\right]}{\beta_{i}^{2} \alpha_{n}(R / a)},
\end{aligned}
$$

$\gamma(x)=\left(3-x^{2}\right) \sin x-3 x \cos x, \alpha_{n} \equiv R \omega_{n} / v_{c}, X_{j}=C_{j}$ for the forcing terms $F_{i}^{\epsilon}$, and $X_{j}=D_{j} / n_{+}^{0}$ for the forcing terms $F_{i}^{Q}$ associated with the $n$th mode of oscillation. The functions $N_{0}, N_{1}$, and $N_{2}$ in Eq. (12c) can be expressed in terms of the equilibrium (spherically symmetric) density profile $n^{0}(r)$ :

$$
\begin{aligned}
& N_{0}(r)=\frac{5}{9} a_{1}\left[n^{0}(r)\right]^{-1 / 3}+a_{2}\left[n^{0}(r)\right]^{-3}\left[\frac{d n^{0}(r)}{d r}\right]^{2}+2 a_{2} \frac{n^{0}(r)}{r^{2}}-\frac{1}{9}\left[n^{0}(r)\right]^{-2 / 3}\left[2 a_{3}+a_{4} a_{5} \frac{\left\{2 a_{5}+\left[n^{0}(r)\right]^{1 / 3}\right\}}{\left\{a_{5}+\left[n^{0}(r)\right]^{1 / 3}\right\}^{3}}\right] \\
& N_{1}(r)=a_{2} n^{0}(r) \\
& N_{2}(r)=-2 a_{2}\left[n^{0}(r)\right]^{-2}\left(\frac{d n_{0}(r)}{d r}\right) .
\end{aligned}
$$

In terms of the above-defined expressions evaluated for the $j$ th phonon mode, the constant $K_{j}\left(\left|\mathbf{p}_{j}\right|=K_{j} Q_{j}\right)$ may be expressed as

$$
K_{j}=n_{+}^{0}\left|\sum_{m, n} F_{m}^{\epsilon}\left(M^{-1}\right)_{m n} F_{n}^{Q}-\frac{4 \pi}{3} R^{3} \frac{\gamma\left(\alpha_{j}\right)}{\alpha_{j}^{3}}\right|
$$

Substitution of Eq. (14) into Eq. (9b) completes the expression for $\left|\mathbf{p}_{j}\right|$.

\section{RESULTS AND DISCUSSION}

For numerical calculation of phonon absorption by small A1 spheres I use for $n_{+}^{0}=\left(\frac{4}{3} \pi r_{s}^{3}\right)^{-1}$ with $r_{s}=2.07$ appropriate for Al. Equilibrium electron distribution is calculated variationally by using the two-parameter trial function

$$
\begin{aligned}
N_{0}(r)=c & \{1+\exp [2 b(r-R-a)] \\
& +\exp [-2 b(r+R+a)]\}^{-1}
\end{aligned}
$$


suggested by Snider and Sorbello, ${ }^{8}$ where $a$ and $b$ are variational parameters and $c$ is a normalization constant determined by the condition that the volume integral of $n_{0}(\mathbf{r})$ is equal to $\frac{4}{3} \pi R^{3} n_{+}^{0}$ for ensuring electrical neutrality. The compressional and shear wave speed of Al used ${ }^{15}$ in the calculation are $6.5 \times 10^{5} \mathrm{~cm} / \mathrm{sec}$ and $3.4 \times 10^{5}$ $\mathrm{cm} / \mathrm{sec}$, respectively. Figure 1 displays the results of numerical evaluations for $\delta s$ and $\delta t$ using thirty basis functions for their expansions, Eqs. (11a) and (11b), with $R=13 \AA$. Good convergence of the result is confirmed by checking the sensitivity of the final answers to the number of basis functions used in the evaluation. In Fig. 1 (a) it is seen that the electron-density response to the electric field exhibits a peak centered about $0.8 \AA$ outside of the sphere boundary. This behavior is similar to that obtained by Snider and Sorbello, ${ }^{8}$ and by Ekardt. ${ }^{9}$ It should be noted that the peak in $\delta n(r)$ is responsible for screening of the electric field from the sphere's interior, and the fact that the peak is outside the jellium sphere boundary indicates that the polarizability of a small metal sphere actually increases with respect to its classical value.

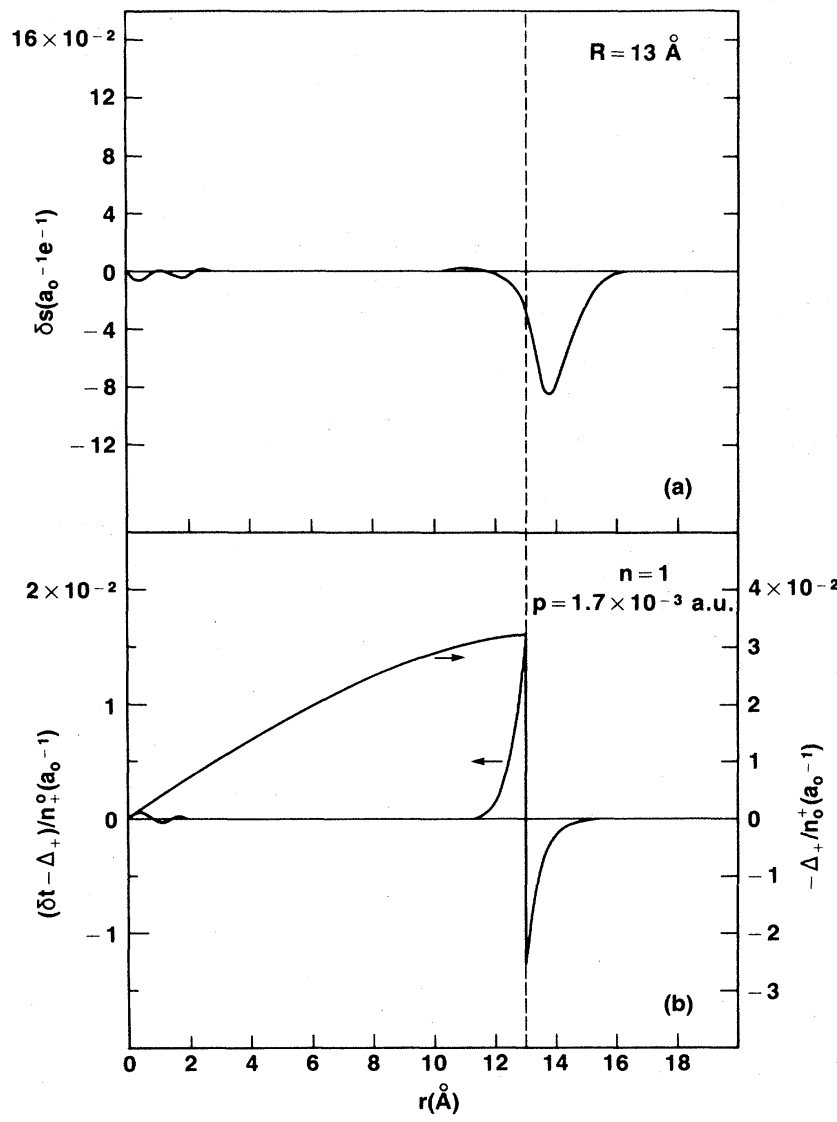

FIG. 1. Electronic response to electric field and phonon oscillation for a sphere with radius $R=13 \AA$. (a) Electron-density change under an electric field. (b) Jellium-density modulation and the residual left after being compensated by the screening electrons. The units of the plotted quantities are in atomic units: $a_{0}$ denotes the Bohr radius, $=0.529 \AA$, and $e$ denotes electronic charge.
The physical basis of the latter result lies in the fact that the jellium Coulomb potential has a "soft" boundary, and the electrons, with their light mass, tend to spill over. As pointed out before, ${ }^{8,9}$ this type of behavior is in sharp contrast to the prediction of those theories using local dielectric function for the metal sphere or infinite potential barrier at the sphere boundary [in terms of dielectric response theory, the evaluation of $\delta n(r)$ in the present formalism is equivalent to the calculation of the static, nonlocal dielectric constant of a metal sphere within the framework of the jellium model]. In Fig. 1(b) one of the curves shows the radial dependence of jellium-density modulation, $\Delta_{+}(r)$, for the $n=1$ normal mode (frequency $=49.3$ $\left.\mathrm{cm}^{-1}\right)$. The other curve gives the radial dependence of the difference between $\Delta_{+}(r)$ and its induced electron-density response $\delta t(r)$. It is seen that whereas deep inside the sphere the electron-density variation closely compensates the jellium-density modulation, near the surface the compensation is not nearly so complete. As a result, there is a surface double layer which, with its dipolar angular dependence (both $\Delta_{+}$and $\delta t$ have $\cos \theta$ dependence), gives rise to two oppositely aligned dipoles of nearly the same magnitude. Evaluation of the net dipole moments for different sphere radii and different normal modes shows that the magnitude of $p$ is nearly a constant as a function of sphere radius but can vary appreciably from one phonon mode to the next. For $R=13 \AA$, dipole moments of the first five modes are $p=1.7 \times 10^{-3}, 1.6 \times 10^{-3}, 1.6 \times 10^{-3}$, $4.9 \times 10^{-4}, 1.2 \times 10^{-3}$ a.u. The especially small value of the $n=4$ mode can be accounted for by the fact that there is a large shear component in its displacement field, which gives no dilatational change of the local volume.

To calculate the far-infrared absorption resulting from the coupling of the dipoles to the electromagnetic radiation, Eq. (10) is used. It should be noted that since the metal particles contain only an integer number $N$ of electrons, the integral in Eq. (10a) should really be expressed as a summation over discrete radii $R_{N}=N^{1 / 3} r_{s}$. For metal particles with electron number distribution given by a discrete log-normal distribution,

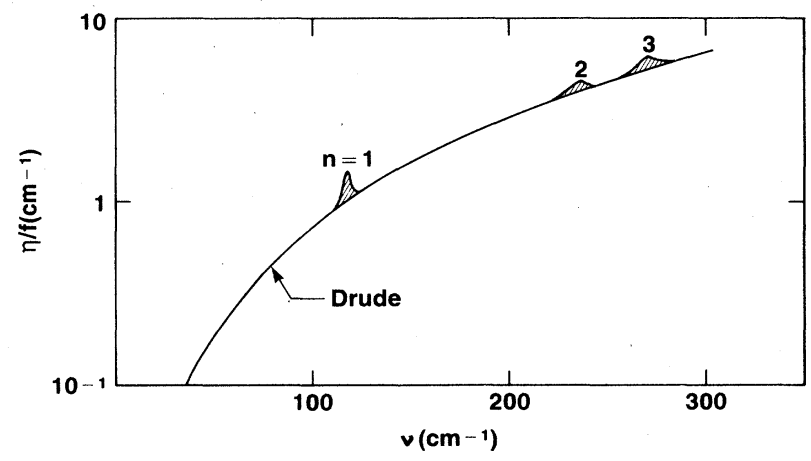

FIG. 2. Frequency variation of the total absorption normalized by the volume fraction of metal particles. Parameters used in the calculation are given in the text. The dipole moments of the three phonon lines are $p_{1}=0.0024, p_{2}=0.0027$, and $p_{3}=0.0037$, in a.u. 


$$
\begin{aligned}
P(N)= & {\left[(2 \pi)^{1 / 2} N \ln \mu\right]^{-1} } \\
& \times \exp \left\{-\left[\ln \left(N / N_{0}\right)\right]^{2} / 2(\ln \mu)^{2}\right\},
\end{aligned}
$$

the frequency dependence of the calculated total absorption (Drude ${ }^{16}$ plus phonon) is plotted in Fig. 2 for temperature $T=0$. In the calculation I have used $N_{0}=125$ $\left(R_{0} \sim 5 \AA\right), \mu=1.05$, i.e., a narrow size distribution, and ascribed a width ${ }^{17} \Gamma_{n}=0.01 v_{n}$ for the phonon-absorption lines. It is seen that the phonon absorption is comparable in magnitude to Drude absorption and is clearly visible against the Drude background (at room temperature the peak should be even higher). This conclusion remains valid even if $\Gamma_{N}$ 's are much larger (e.g., by a factor of 3). However, the phonon absorption would become smeared and submerged in the Drude absorption for a broad size distribution, e.g., $\mu=1.6$. This fact emphasizes the importance of well-defined particle sizes for the experimental observation of phonon absorption. For particles larger than $10 \AA$ ( $5 \AA$ radius), the fact that the dipole moments are roughly constant means that the absorption constant has a $R_{0}^{-3}$ size dependence as can be inferred from Eq. (10). This restricts the observation of phonon absorption to particle systems with $R_{0} \leqq 10 \AA$ (20 $\AA$ particles or smaller).

In summary, calculation within the quasistatic jellium model has shown significant coupling between the phonons of metal particles $<20 \AA$ in diameter and the electromagnetic radiation. It is proposed that experimentally these phonon-absorption lines not only could serve as a probe of particle structure and possible lattice softening effects, but their observation (or nonobservation) under controlled conditions may also help to elucidate the origin of the absorption enhancement effect at infrared frequencies. $^{1,2}$

\section{ACKNOWLEDGMENTS}

I wish to acknowledge many helpful discussions with $\mathrm{T}$. Upton, R. Tao, and J. Bernholc.
${ }^{1}$ R. P. Devaty and A. J. Sievers, Phys. Rev. Lett. 52, 1344 (1984).

${ }^{2}$ N. E. Russell, J. C. Garland, and D. B. Tanner, Phys. Rev. B 23, 632 (1981).

${ }^{3}$ H. Chew, P. J. McNulty, and M. Kerker, Phys. Rev. A 13, 396 (1976).

${ }^{4}$ D. A. Weitz, T. J. Gramila, A. Z. Genack, and J. I. Gersten, Phys. Rev. Lett. 45, 355 (1980).

5P. F. Liao, J. G. Bergman, D. S. Chemla, A. Wokan, J. Melngailis, A. M. Hawryluk, and N. P. Economou, Chem. Phys. Lett. 82, 355 (1981).

6P. Apell and D. R. Penn, Phys. Rev. Lett. 50, 1316 (1983).

${ }^{7}$ D. M. Wood and N. W. Ashcroft, Phys. Rev. B 25, 6255 (1982).

${ }^{8}$ D. R. Snider and R. S. Sorbello, Phys. Rev. B 28, 5702 (1983).

${ }^{9}$ W. Ekardt, Phys. Rev. Lett. 52, 1925 (1984).

${ }^{10}$ B. B. Dasgupta and R. Fuchs, Phys. Rev. B 24, 554 (1981).

${ }^{11}$ A. J. Glick and E. D. Yorke, Phys. Rev. B 18, 2490 (1978).

${ }^{12} \mathrm{~W}$. Kohn and P. Vashishta, in Theory of the Inhomogeneous Electron Gas, edited by S. Lundquist and N. H. March (Plenum, New York, 1983), p. 75.
13J. M. Ziman, Principles of the Theory of Solids (Cambridge University Press, London, 1964), p. 219. See also R. Landauer, in Electrical Transport and Optical Properties of Inhomogeneous Media, edited by J. C. Garland and D. B. Tanner (AIP, New York, 1978).

${ }^{14}$ P. M. Morse and H. Feshbach, Methods of Theoretical Physics (McGraw-Hill, New York, 1953), Vol. II, p. 1759. It should be noted that the traction formula $(13.3 .78)$ on p. 1872 contains an error of 2 in one of its factors.

${ }^{15}$ C. Kittel, Introduction to Solid State Physics, 3rd edition (John Wiley \& Sons, Inc., New York, 1966), p. 122.

${ }^{16}$ Here I have included both the electric and magnetic dipole terms and assumed that the relaxation time $\tau$ is given by $\tau=R / V_{F}$. See D. Stroud and F. P. Pan, Phys. Rev. B 17, 1602 (1978). For $R=5 \AA$ the magnetic dipole term is actually negligible.

${ }^{17}$ The assumed phonon-absorption linewidth, $\Gamma_{n}=0.01 v_{n}$, is in accord with the observed Reststrahlen absorption width in bulk crystals. See, for example, C. F. Bohren and D. R. Huffman, Absorption and Scattering of Light by Small Particles (John Wiley, New York, 1983), pp. 241-251. 